\title{
SUBCATEGORIES OF POSITIVE EMOTION
}

\author{
Tomomi FUJIMURA ${ }^{1,2)}$, Wataru SATO ${ }^{3)}$, and Kazuo OKANOYA ${ }^{1,2), 4)}$ \\ 1) Japan Science Technology Agency, ERATO, Japan \\ ${ }^{2)}$ RIKEN Brain Science Institute, Japan \\ ${ }^{3)}$ Kyoto University, Japan \\ ${ }^{4)}$ The University of Tokyo, Japan
}

\begin{abstract}
Positive emotions are indispensable in our lives. However, their structure has not yet been clarified, and the majority of previous studies have simply assumed a single category of positive emotions. Several recent psychological and neuroscientific findings suggest the existence of subcategories of positive emotion. Similarly, the psychological functions of positive emotions can be more deeply understood when they are subcategorized. We conclude that subcategorizing positive emotions is a promising avenue for future research on emotion.
\end{abstract}

Key words: positive emotions, subcategories, psychological functions

Positive emotions enrich our lives. We experience them in situations such as eating delicious food, seeing lovely babies, and watching funny TV programs. There are many words describing positive emotions, such as contentment, amusement, and interest. We can easily distinguish these emotions based on everyday experience.

However, the structure of positive emotion has not yet been clarified by scientific research. In the majority of previous psychological studies on emotion, positive emotions have been represented as a single category such as "happy" or "pleasant" (Ekman, 1992). This contrasts with negative emotions, for which several subcategories have been proposed (e.g., anger, sadness, disgust, and fear) (Ekman, 1992).

A few recent studies have addressed the presence of subcategories of positive emotion (Fujimura, Sato, \& Suzuki, 2010; Fujimura \& Suzuki, 2010; Takahashi et al., 2008). In this review, we discuss these subcategories. First, we outline how positive emotions were described in the previous major theories of emotion. Then, we introduce new psychological and neuroscientific findings supporting the presence of the subcategories. Finally, we discuss psychological functions of subcategorized positive emotions.

\section{Positive Emotions in Previous Theories}

Previous theories of emotion can be generally classed into categorical and dimensional theories (Christie \& Friedman, 2004; Fujimura, Matsuda, Katahira, Okada, \&

This study was supported by the JSPS Funding Program for Next Generation World-Leading Researchers.

Correspondence concerning this article should be addressed to Wataru Sato, The Hakubi Project, Primate Research Institute, Kyoto University, Inuyama, Aichi 484-8506, Japan (e-mail: sato@pri.kyoto-u.ac.jp). 
Okanoya, 2011; Panayiotou, 2008).

The categorical or basic emotion theory has been one of the most common (Ekman, 1984, 1992; Izard, 1991; Johnson-Laird \& Oatley, 1992; Plutchik, 1980). This theory asserts the existence of basic emotions that have corresponding and distinct facial expressions and physiological reactions. There are several viewpoints regarding the number of basic emotions, but six categories are most frequently accepted: happiness, surprise, disgust, anger, fear, and sadness (Ekman, 1972; Ekman \& Friesen, 1976). Obviously, the six basic emotions include only one positive emotion, happiness. Is the structure of positive emotion really so simple? Even Ekman, who proposed the six basic emotions, stated: "I believe that there are more than a dozen enjoyable emotions, each universal, each as different from the other as sadness, anger, fear, disgust, and contempt are from one another."(Ekman, 2003). It seems likely that positive emotions can be categorized in a similar way to negative ones.

The other model of emotion, the dimensional theory, asserts that emotions are continuously represented in a space consisting of two dimensions, valence and arousal (Russell, 1980; Russell \& Bullock, 1985). The exact number and labeling of dimensions vary among studies, but valence is regarded as the primary dimension in most of them. The valence dimension is bipolar: positivity/pleasantness vs. negativity/unpleasantness. Positive and negative emotions are represented by quantitative variation in the valence dimension. However, positive emotions are still not distinguished into subcategories.

In sum, previous major theories have not referred to subcategories of positive emotion.

\section{Psychological Evidence for SubCategories of Positive Emotion}

Two recent psychological studies on facial expressions have provided evidence for differentiated positive emotions (Fujimura et al., 2010; Fujimura \& Suzuki, 2010).

Fujimura and Suzuki (2010) investigated recognition of various negative and positive emotions using both dynamic and static facial expressions. The facial stimuli were based on the dimensional viewpoint and included three positive facial expressions: excitement, happiness, and calmness, according to arousal level. These emotion category labels were arbitrarily assigned from high-arousal to low-arousal expressions (Russell, 1997). Participants were asked to rate static and dynamic facial stimuli using a forcedchoice task in which they classified facial stimuli into emotional categories. The results showed that dynamic excited and happy faces had higher recognition rates than static faces (see Fig. 1). Furthermore, although the recognition rate for static excited faces was below chance (i.e., 33\%), that for dynamic excited face exceeded chance. The data indicate that positive facial expressions are not solely attributed to happiness or pleasantness, but can be more differentiated with a dynamic presentation. Emotional categorization of positive facial stimuli was clearer in the dynamic presentation condition than in the static presentation condition. Compared to static facial photos, dynamic facial expressions are more natural and powerful, and reveal psychological processes more 


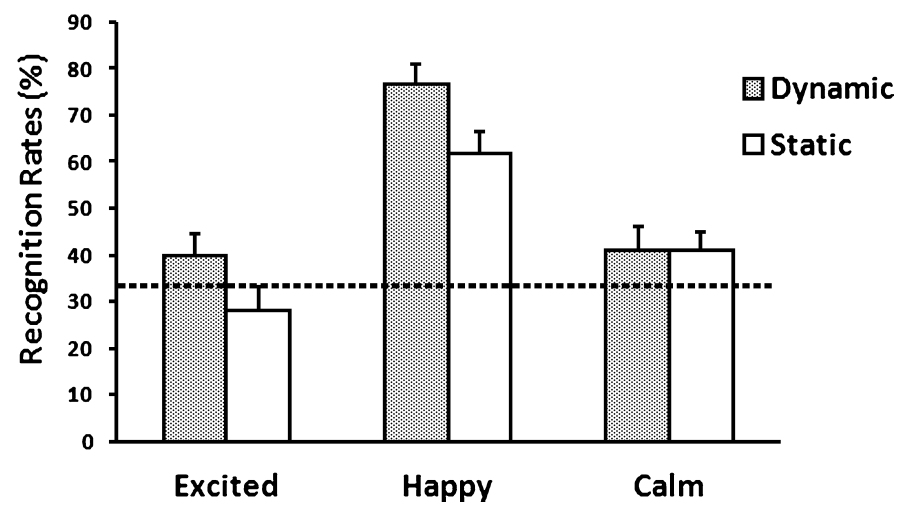

Fig. 1. Mean $(S E)$ recognition rates for positive emotions in the forced-choice task in Fujimura and Suzuki (2010). Dynamic excited and calm faces produced higher recognition rates than static ones. The dotted line indicates chance level (i.e., 33\%). The recognition rate for dynamic excited faces exceeds chance.

realistically (Sato \& Yoshikawa, 2007). Hence, the evidence for differentiated positive emotions in these studies is likely to be more valid than previous studies using static stimuli.

Dynamic positive expressions are also discriminated based on genuineness. Krumhuber and Kappas (2005) tested the effect of temporal dynamics of smiles on the perception of smile authenticity. The results showed that smiles with a longer onset or offset time were judged as more genuine than those with a shorter duration. Temporal dynamics influence the perceived genuineness of smiles, indicating that people can discriminate characteristics of positive emotional expressions.

Fujimura et al. (2010) tested facial mimicry, providing further evidence for the presence of subcategories of positive emotion. Facial mimicry involves automatic facial reactions corresponding to observed facial expressions (Hess, Philippot, \& Blairy, 1999). Unconscious smiles in response to another's smile promote smooth communication between individuals. Previous psychophysiological studies using facial electromyography (EMG) have reported that facial mimicry occurs automatically in response to facial expressions (Dimberg, 1982; Dimberg \& Thunberg, 1998; Dimberg, Thunberg, \& Elmehed, 2000). For example, Dimberg (1982) showed that mere photographic presentations of angry and happy facial expressions induced spontaneous corrugator supercilii muscle activity (brow lowering actions, prototypical in angry facial expressions) and zygomatic major muscle activity (lip corner pulling actions, prototypical in happy facial expressions), respectively. Fujimura et al. (2010) recorded facial EMG while participants observed the dynamic and static facial expressions of two positive emotions, excitement and calm, and two negative emotions. The results revealed that zygomatic major muscle activity was stronger in response to excited faces than to calm faces. It is possible that facial mimicry is based on greater differentiation between positive emotional expressions than simply smiling. 
Facial mimicry also reflects authenticity, represented by subtle differences in others' positive facial expressions. Surakka and Hietanen (1998) investigated zygomatic and orbicularis oculi muscle activities in response to a Duchenne-smile with orbicularis oculi muscle activity, which is called a true smile, and a non-Duchenne smile without such muscle activity. The EMG activities of zygomatic and orbicularis oculi were significantly higher for the Duchenne-smile than for neutral facial expressions, but there was no significant difference between the response to the non-Duchenne smile and neutral expressions. This indicates that the subtle featural differences of positive expressions are reflected in observers' facial reactions. That is, differentiated positive expressions can be detected and reflected in facial mimicry.

In summary, these data suggest that humans do not simply interpret positive facial expressions as happiness and respond to them; they perceive various types of positive emotions expressed by others. That is, humans are more sensitive to subcategories of positive expression than previous psychological studies have suggested.

\section{Neuroscientific Evidence for SubCAtegories of Positive Emotion}

Several previous neuroimaging studies have investigated neural substrates of positive emotions using various types of stimuli, such as sexually arousing scenes (Arnow et al., 2002), happy facial expressions (Sato, Kochiyama, \& Yoshikawa, 2010), and pleasant flavors (de Araujo, Rolls, Kringelbach, McGlone, \& Phillips, 2003). These studies have shown that stimuli that elicit positive emotions activate brain regions such as the nucleus accumbens, amygdala, orbitofrontal cortex, and anterior cingulate cortex (for a review, see Liu, Hairston, Schrier, \& Fan, 2011). However, most studies have not tested more than two types of positive emotions.

A recent neuroimaging study investigated brain activity during two types of positive emotions: the basic positive emotion, joy, and the socially positive emotion, pride (Takahashi et al., 2008). Participants were presented with sentences describing emotional situations, and asked to imagine that the scenario protagonist was himself/herself. The joy condition included sentences such as, "I had a delicious dinner", and the pride condition included sentences such as, "I graduated at the head of my class". The results showed that experiencing joy activated brain regions such as the nucleus accumbens and anterior cingulate cortex, whereas experiencing pride activated different regions in the posterior superior temporal sulcus and temporal pole. These data indicate that the two types of positive emotions, joy and pride, have different neural underpinnings and suggest the presence of subcategorized positive emotion at a neural level.

\section{Functions of SubCategorized Positive Emotions}

It is important to subcategorize positive emotions in order to understand the function

of positive emotions. Positive emotions have been shown to have adaptive psychological 
functions. In a review of the literature, Fredrickson (2001) proposed the broaden-andbuild theory of positive emotions. She suggests that positive emotions broaden one's repertoire of thoughts and behaviors, and increase the flexibility of one's perceptual and behavioral patterns, which, in turn, builds enduring personal resources, such as mindfulness, resilience, social closeness, and even physical health (Fredrickson, 2001; Garland, Fredrickson, Kring, Johnson, Meyer, \& Penn, 2010).

Increasing creativity thus appears to be one of the functions of positive emotions. Several previous studies have shown that induction of positive emotions facilitates creative thinking (Isen, Daubman, \& Nowicki, 1987). However, a recent meta-analysis (Baas, De Dreu, \& Nijstad, 2008) has reported that activated positive emotions (e.g., happiness) increase creativity, while deactivated positive emotions (e.g., relaxation) do not. These data indicate that only a subset of positive emotions support the broaden-andbuild theory. This suggests that subcategorized positive emotions may have different psychological functions.

Another line of research has indicated that positive emotions facilitate recovery from the cardiovascular activation induced by negative emotion (Fredrickson \& Levenson, 1998; Fredrickson, Mancuso, Branigan, \& Tugade, 2000). This is referred to as an "undoing effect". As chronic physiological arousal elicited by negative emotion can lead to psychosomatic disorders and depression (Gotlib \& Joormann, 2010), positive emotions appear to protect psychological health. However, it is unknown whether all positive emotions similarly exhibit such an effect. In a previous study on this issue, researchers tested contentment and amusement, and found no significant difference in the undoing effect (Fredrickson \& Levenson, 1998); however, these results cannot be generalized to other positive emotions. Compared to these two emotions, excitement and calm, for example, are respectively more and less arousing. As in the case of Fujimura et al.'s (2010) facial EMG study, the use of these emotions may reveal different psychophysiological effects.

Positive emotions have also been shown to modulate perception. For example, positive emotions lead to increased global, but not local, visual processing (Fredrickson \& Branigan, 2005) and positive emotions impair selective visual attention by increasing the processing of spatially adjacent stimuli (Rowe, Hirsh, \& Anderson, 2007). It has been suggested that this is due to a broadening effect on cognition, and that positive emotions enable an increased incorporation of external information. Moreover, positive emotions facilitate the context effect in perception (Avramova, Stapel, \& Lerouge, 2010). As proposed by the broaden-build theory, positive emotions appear to enhance the tendency to incorporate surrounding and contextual information. However, these studies examined a single category of positive emotion and did not assume subcategorized positive emotions.

There is not sufficient evidence to conclude that all positive emotions increase flexibility of thought and broaden behavioral repertoires. Further studies are necessary to test various types of positive emotions and compare the effect of differentiated positive emotions. The finding that subcategories of positive emotion have specific psychological functions would deepen our understanding of the structure and the function of emotion. 


\section{LIMITATIONS AND FUTURE DIRECTIONS}

This review presented psychological and neuroscientific evidence suggesting the existence of subcategories of positive emotion. Subcategories of positive emotion provide a new framework for understanding the structure and the function of emotion. However, some issues remain to be clarified in future research.

The first issue concerns how to subcategorize positive emotions. The psychological studies of facial expressions (e.g., Fujimura \& Suzuki, 2010) and creativity (e.g., Baas et al., 2008) provided evidence for subcategorized positive emotions based on arousal level, whereas the neuroimaging study (Takahashi et al., 2008) showed different neural underpinnings of social and non-social emotions. Arousal level and the social nature of emotions are two criteria used to subcategorize positive emotions; however, it is not clear how these criteria are related to each other. The emotions differentiated by arousal level, excitement, happiness, and calmness are more basic than social emotions such as pride because arousal is a fundamental component of the emotional space (Russell, 1980; Russell \& Bullock, 1985). Future research must clarify the relationship between basic and social emotions in order to develop a model to explain the structure of positive emotion. Furthermore, studies that have provided evidence for subcategories of positive emotion have used widely differing stimuli such as facial expressions (e.g., Fujimura \& Suzuki, 2010) and sentences expressing emotional content (Takahashi et al., 2008); thus, future investigations of positive emotion subcategories should use a common methodology.

Second, the relationship between the subcategories of positive emotions and those of negative emotions (i.e., anger, fear, and sadness) is not yet defined. From the viewpoint of the dimensional theory, subcategories of positive and negative emotions may be qualitatively equivalent because both are represented as differences in arousal level (Russell, 1980; Russell \& Bullock, 1985). In the two-dimensional emotional space, positive emotions are compatible with negative emotions. However, it is not clear whether subcategories of positive emotion are represented at the same level as those of negative emotions within the basic emotion theory (Ekman, 1984; Ekman, 1992).

We propose two possible directions for future studies of the relationship between the subcategories of positive and negative emotions. The first is the investigation of whether facial expressions produced by subcategorized positive emotions can be identified crossculturally. Individuals in Western and non-Western cultures can identify six basic emotions (i.e., happiness, anger, sadness, fear, disgust, and surprise) (Ekman et al., 1987). If subcategorized positive emotions were basic, the facial expressions of these positive emotions would show cross-cultural agreement. A second line of investigation is whether subcategories of positive emotion have specific autonomic activities. Negative emotions have been shown to have distinctive patterns of autonomic nervous system activity (Ekman, Levenson, \& Friesen, 1983; Levenson, Ekman, \& Friesen, 1990), but the autonomic responses evoked by positive emotions are less well known. A recent psychophysiological study reported that the heart rate deceleration pattern elicited by amusement differed from that elicited by joy (Herring, Burleson, Roberts, \& Devine, 2011). It is possible that subcategorized positive emotions also show distinctive autonomic activity. 


\section{CONCLUSION}

Positive emotions are indispensable in our lives. We label them in diverse ways: amusement, joy, contentment, elation, and pride. However, the structure of positive emotion and the functions of their subcategories have been left little investigated. Recent psychological and neuroscientific studies (Fujimura et al., 2010; Fujimura \& Suzuki, 2010; Takahashi et al., 2008) have suggested the presence of subcategories of positive emotion. Similarly, we suggest that the psychological functions of positive emotions can be more deeply understood when they are subcategorized. It has not been long since the field of positive psychology emerged. Subcategorizing positive emotions in more detail will provide us with clues to elucidate the benefits of positive emotions for individuals and society.

\section{REFERENCES}

Arnow, B. A., Desmond, J. E., Banner, L. L., Glover, G. H., Solomon, A., Polan et al. 2002. Brain activation and sexual arousal in healthy, heterosexual males. Brain, 125, 1014-1023.

Avramova, Y. R., Stapel, D. A., \& Lerouge, D. 2010. Mood and context-dependence: Positive mood increases and negative mood decreases the effects of context on perception. Journal of Personality and Social Psychology, 99, 203-214.

Baas, M., De Dreu, C. K., \& Nijstad, B. A. 2008. A meta-analysis of 25 years of mood-creativity research: hedonic tone, activation, or regulatory focus? Psychological Bulletin, 134, 779-806.

Christie, I., \& Friedman, B. H. 2004. Autonomic specificity of discrete emotion and dimensions of affective space: a multivariate approach. International Journal of Psychophysiology, 51, 143-153.

de Araujo, I. E., Rolls, E. T., Kringelbach, M. L., McGlone, F., \& Phillips, N. 2003. Taste-olfactory convergence, and the representation of the pleasantness of flavour, in the human brain. European Journal of Neuroscience, 18, 2059-2068.

Dimberg, U. 1982. Facial reactions to facial expressions. Psychophysiology, 19, 643-647.

Dimberg, U., \& Thunberg, M. 1998. Rapid facial reactions to emotional facial expressions. Scandinavian Journal of Psychology, 39, 39-45.

Dimberg, U., Thunberg, M., \& Elmehed, K. 2000. Unconscious facial reactions to emotional facial expressions. Psychological Science, 11, 86-89.

Ekman, P. 1972. Universal and cultural differences in facial expressions of emotion. In K. Cole (Ed.), Nebraska symposium on motivation (Vol. 19, pp. 207-283). Lincoln, NE: University of Nebraska Press.

Ekman, P. 1984. Expression and the nature of emotion. In P. Ekman \& K. R. Scherer (Eds.), Approaches to emotion. (pp. 319-343). Hillsdale, NJ: Lawrence Erlbaum Association, Inc.

Ekman, P. 1992. An argument for basic emotions. Cognition \& Emotion, 6, 169-200.

Ekman, P. 2003. Emotion Revealed. New York: Times Books.

Ekman, P., \& Friesen, W. V. 1976. Pictures of facial affect. Palo Alto, California: Consulting Psychologists Press.

Ekman, P., Friesen, W. V., O'Sullivan, M., Chan, A., Diacoyanni-Tarlatzis, I., Heider, K. et al. 1987. Universals and cultural differences in the judgments of facial expressions of emotion. Journal of Personality and Social Psychology, 53, 712-717.

Ekman, P., Levenson, R. W., \& Friesen, W. V. 1983. Autonomic nervous system activity distinguishes among emotions. Science, 221, 1208-1210.

Fredrickson, B. L. 2001. The role of positive emotions in positive psychology: The broaden-and-build theory of positive emotions. American Psychologist, 56, 218-226.

Fredrickson, B. L., \& Branigan, C. 2005. Positive emotions broaden the scope of attention and thought action repertoires. Cognition \& Emotion, 19, 313-332. 
Fredrickson, B. L., \& Levenson, R. W. 1998. Positive emotions speed recovery from the cardiovascular sequelae of negative emotions. Cognition \& Emotion, 12, 191-220.

Fredrickson, B. L., Mancuso, R. A., Branigan, C., \& Tugade, M. M. 2000. The undoing effect of positive emotions. Motivation and Emotion, 24, 237-258.

Fujimura, T., Matsuda, Y., Katahira, K., Okada, M., \& Okanoya, K. 2011. Categorical and dimensional perceptions in decoding emotional facial expressions. Cognition \& Emotion. [Epub ahead of print]

Fujimura, T., Sato, W., \& Suzuki, N. 2010. Facial expression arousal level modulates facial mimicry. International Journal of Psychophysiology, 76, 88-92.

Fujimura, T., \& Suzuki, N. 2010. Effects of dynamic information in recognising facial expressions on dimensional and categorical judgments. Perception, 39, 543-552.

Garland, E. L., Fredrickson, B., Kring, A. M., Johnson, D. P., Meyer, P. S., \& Penn, D. L. 2010. Upward spirals of positive emotions counter downward spirals of negativity: insights from the broaden-andbuild theory and affective neuroscience on the treatment of emotion dysfunctions and deficits in psychopathology. Clinical Psychology Review, 30, 849-864.

Gotlib, I. H., \& Joormann, J. 2010. Cognition and depression: current status and future directions. Annual Review of Clinical Psychology, 6, 285-312.

Herring, D. R., Burleson, M. H., Roberts, N. A., \& Devine, M. J. 2011. Coherent with laughter: subjective experience, behavior, and physiological responses during amusement and joy. International Journal of Psychophysiology, 79, 211-218.

Hess, U., Philippot, P., \& Blairy, S. 1999. Mimicry: Fact and fiction. In P. Philippot, R. S. Feldman \& E. J. Coats (Eds.), The social context of nonverbal behavior (pp. 213-241). New York: Cambridge University Press.

Isen, A. M., Daubman, K. A., \& Nowicki, G. P. 1987. Positive affect facilitates creative problem solving. Journal of Personality and Social Psychology, 52, 1122-1131.

Izard, C. E. 1991. The psychology of emotions. New York: Plenum.

Johnson-Laird, P. N., \& Oatley, K. 1992. Basic emotions, rationality, and folk theory. Cognition \& Emotion, 6, 201-223.

Krumhuber, E., \& Kappas, A. 2005. Moving smiles: The role of dynamic components for the perception of the genuineness of smiles. Journal of Nonverbal Behavior, 29, 3-24.

Levenson, R. W., Ekman, P., \& Friesen, W. V. 1990. Voluntary facial action generates emotion-specific autonomic nervous system activity. Psychophysiology, 27, 363-384.

Liu, X., Hairston, J., Schrier, M., \& Fan, J. 2011. Common and distinct networks underlying reward valence and processing stages: a meta-analysis of functional neuroimaging studies. Neuroscience and Biobehavioral Reviews, 35, 1219-1236.

Panayiotou, G. 2008. Emotional dimensions reflected in ratings of affective scripts. Personality and Individual Differences, 44, 1795-1806.

Plutchik, R. 1980. Emotions: A psychoevolutionary synthesis. New York: Harper \& Row.

Rowe, G., Hirsh, J. B., \& Anderson, A. K. 2007. Positive affect increases the breadth of attentional selection. Proceedings of the National Academy of Sciences of the United States of America, 104, 383-388.

Russell, J. A. 1980. A circumplex model of affect. Journal of Personality and Social Psychology, 39, 11611178.

Russell, J. A. 1997. Reading emotions from and into faces. In J. A. Russell \& J. M. Fernändez-Dols (Eds.), The Psychology of Facial Expression (pp. 295-320). Paris: Cambridge University Press.

Russell, J. A., \& Bullock, M. 1985. Multidimensional scaling of emotional facial expressions: Similarity from preschoolers to adults. Journal of Personality and Social Psychology, 48, 1290-1298.

Sato, W., Kochiyama, T., \& Yoshikawa, S. 2010. Amygdala activity in response to forward versus backward dynamic facial expressions. Brain Research, 1315, 92-99.

Sato, W., \& Yoshikawa, S. 2007. Spontaneous facial mimicry in response to dynamic facial expressions. Cognition, 104, 1-18.

Surakka, V., \& Hietanen, J. K. 1998. Facial and emotional reactions to Duchenne and non-Duchenne smiles. International Journal of Psychophysiology, 29, 23-33.

Takahashi, H., Matsuura, M., Koeda, M., Yahata, N., Suhara, T., Kato, M. et al. 2008. Brain activations during judgments of positive self-conscious emotion and positive basic emotion: pride and joy. Cerebral Cortex, 18, 898-903. 